\title{
Acute Myocardial Infarction of Embolic Origin, Secondary to Short Circuit Left to Right
}

\author{
Botti Sequera A, Delgado Leal L*, Rodríguez R, Marin D, Lugo R, Hernández J and Salas J \\ Cardiology Department, Mexico \\ *Corresponding author: Delgado Leal L, Cardiology Department, Centenario Hospital Míguel Hidalgo Aguascalientes, \\ Ags. Mexico
}

\section{ARTICLE INFO}

Received: September 13, 2019

Published: 幽 September 19, 2019
ABSTRACT

Citation: Botti Sequera A, Delgado Leal L, Rodríguez R, Marin D, Lugo R, et al., Acute Infarction of The Secondary Embòlical Origin of Secondary Short Circuit Left-Right. Biomed J Sci \& Tech Res 21(3)-2019. BJSTR. MS.ID.003603.

\section{Introduction}

Coronary embolism is a rare cause of acute myocardial infarction (AMI). The prevalence of this non-atherosclerotic entity remains unknown due to its difficult diagnosis in the acute context. In general, 4 to $7 \%$ of all patients diagnosed with AMI do not have atherosclerotic coronary disease at autopsy or coronary angiography. There are several case reports of AMI secondary to thromboembolism. In these reports, valvular atrial fibrillation (no) is the most frequent thromboembolic risk factor. Although coronary embolism is uncommon, there are several causes of coronary artery thromboembolism. Procoagulable states such as obesity, pregnancy, hereditary thrombophilic disorders and cancer are important additional risk factors for coronary embolism [1]. Rarely, a paradoxical embolism that causes acute myocardial infarction (AMI) has been reported in the context of patent foramen ovale (FOP) or atrial septum defect (ASD). It may be related to the presence of right-to-left intracardiac shunt in the context of the hypercoagulable state. Paradoxical embolism due to FOP or ASD that caused AMI has been reported in the literature, but is rarely considered in clinical practice. Coronary embolism should always be suspected in the context of sudden chest pain in patients with valvular prostheses, chronic atrial fibrillation, dilated cardiomyopathy, infective endocarditis, intracardiac shunts, cardiac myxoma, wall thrombi and hypercoagulable states. [2].

\section{Clínic Case}

32-year-old woman, sedentary. History of bronchial asthma in childhood. Gynecological history: Pregnancies 5, Abortions 2, Births 2, Caesarean sections 1. Congenital heart disease diagnosed in childhood, without medical treatment. Chronic active smoking, smoking rate 10 packages / year. Obesity grade III. (Figure1) No history of diabetes mellitus or high blood pressure. She begins suffering two weeks before medical contact with typical angina and decreased functional class, palpitations and lower limb edema. 3 hours prior to admission with 10/10 angina, feeling of imminent death and sudden death that required CPR during their transfer to the emergency service of the Centernario Hospital Miguel Hidalgo. Upon admission he is found with $70 / 40 \mathrm{mmHg}$ blood pressure, tissue hypoperfusion, 70\% oxygen saturation, advanced airway management and invasive mechanical ventilation. EKG is performed in which it is found with atrial fibrillation rhythm, righthand QRS electrical axis, with full block image of the right bundle of the His bundle, ST DII-DIII-aVF elevation, ST elevation in DI and AVL , making a diagnosis of infarction with ST elevation in the lower face.

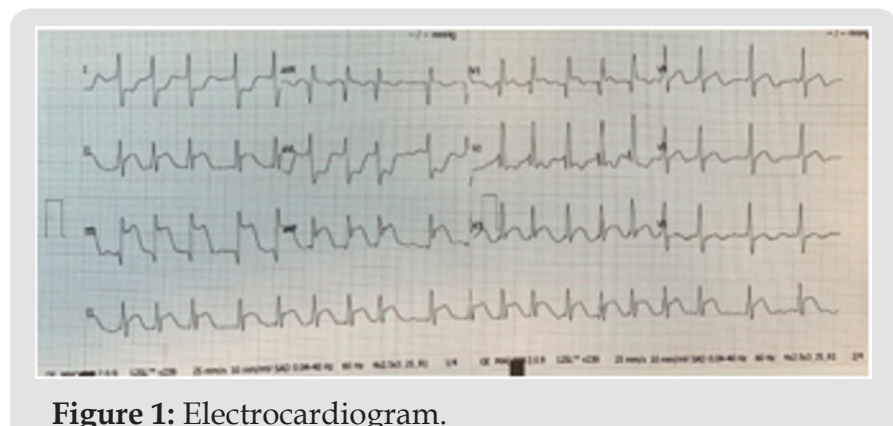

Figure 1: Electrocardiogram.

Electrocardiogram with right leads shows ST segment elevation in V4 (Figures 2 \& 3). ACTP is performed by total occlusion in the distal 
third of the right coronary artery, without atherosclerotic lesions, other normal-looking coronary vessels without atherosclerotic lesions. Upon admission to the coronary intensive care unit, he is found with the following physical examination: Hemodynamic with blood pressure $80 / 40 \mathrm{~mm} \mathrm{Hg}$, with norepinephrine at 0.05gammas and dobutamine at 5 gammas. Cardiovascular, neck with jugular engorgement grade II, no carotid murmurs, with apical impulse in sixth left intercostal space, anterior axillary line, high parasternal lift, Chavez integrates suggestive of pulmonary hypertension, paradoxical splitting of second pulmonary noise. Puff with pulmonary epicenter, mesosystolic, rough, intensity IV / VI, long duration, rough-blowing quality. Mesothelediastolic Erb point (accessory aortic), grade III / VI, blowing quality. Puff with tricuspid epicenter, holosystolic, intensity III / VI, regurgitant, blowing quality. I blow with mitral epicenter, holosystolic, grade II / VI, regurgitant, blowing quality, short duration. Respiratory without rales, crackling. Globose abdomen at the expense of adipose panicle, with no evidence of peritoneal irritation. Lower extremities with edema $+++/+++$, filiform pulse in 4 strictures.

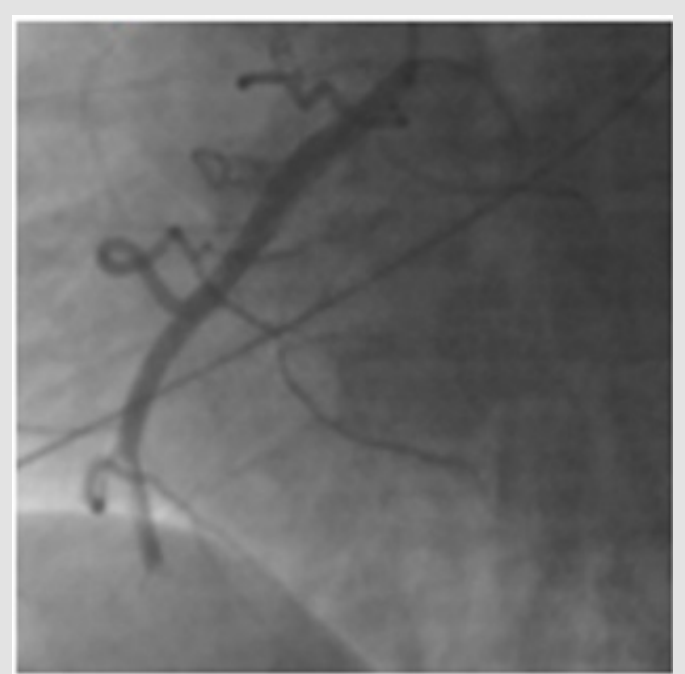

Figure 2: Coronory angiography.

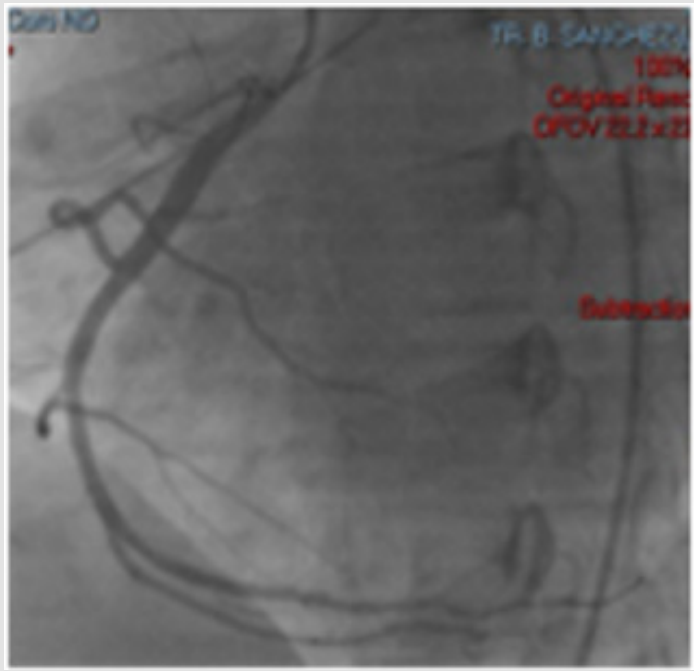

Figure 3: percutaneous coronary intervention.

\section{Diagnostic Aids}

Transesophageal echocardiogram: Severe biatrial dilatation, septum with interatrial communication (ostium secundum), short left-right circuit, Qp-Qs 3.3. Left ventricle without alterations of segmental mobility with mild dilation, left ventricular ejection fraction $45 \%$, right ventricle with hypokinesia, TAPSE 10 , severe tricuspid insufficiency secondary to right ventricular dilation, moderate mitral regurgitation to left ventricular dilation.

\section{Conclusion}

With the physical examination and the previous auxiliary studies, an acute myocardial infarction with electrical extension and hemodynamics is integrated into the right ventricle, in a LUPI C, secondary to a short circuit left to right. Ostium secundum interatrial communication. Receiving infarction treatment with extension to the right ventricle leaving home 2 weeks later, in functional class II / IV. To initiate surgical closure protocol for interatrial septum defect. Acute myocardial infarction of cardioembolic origin is a cause of acute myocardial infarction, in the context of our patient it has multiple risk factors such as atrial fibrillation, obesity and a structural defect of the interatrial septum causing atrial dilation and a Consecutive atrial fibrillation, which presented a heart attack in the territory of the right coronary artery with electrical and hemodynamic extension to the right ventricle, having a favorable outcome for life and function due to its early recognition and immediate management. Acute myocardial infarction of embolic etiology should be suspected in all young patients without cardiovascular risk factors such as diabetes mellitus or arterial hypertension and presenting risk factors such as atrial fibrillation, structural defects and procoagulant conditions in the context of a patient with typical angina that is presented requesting medical attention in the emergency room.

\section{References}

1. C Camaro, Aengevaeren WR (2009) Acute myocardial infarction due to coronary artery embolism in a patient with atrial fibrillation. Neth Heart J 17(8): 297-279.

2. Abdelrahman Jamie, Ahmed Alsaileek, Kamal Ayoub, Ahmad Omran (2012) Paradoxical Embolism in Acute Myocardial Infarction in a Patient with Congenital Heart Disease, Heart Views 13(3): 111-113. 
ISSN: 2574-1241

DOI: 10.26717/BJSTR.2019.21.003603

Delgado Leal L. Biomed J Sci \& Tech Res

(c) (P) This work is licensed under Creative

Submission Link: https://biomedres.us/submit-manuscript.php

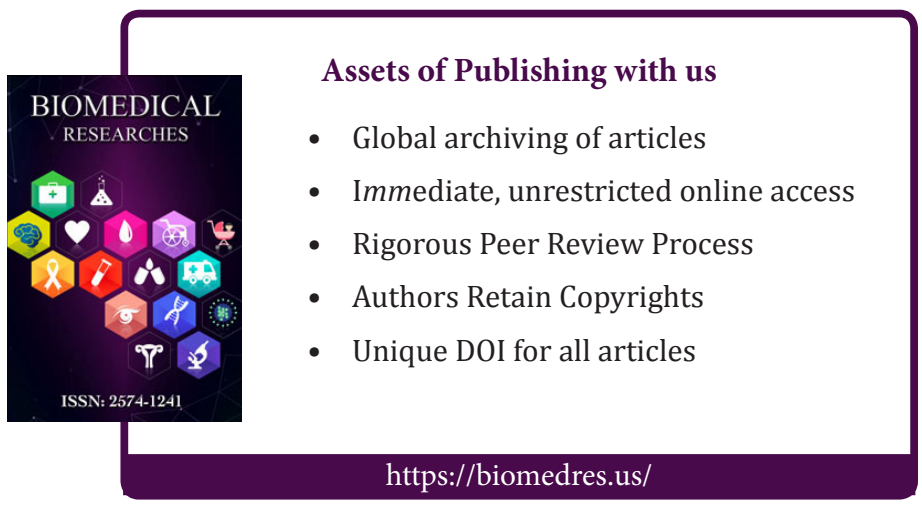

